\title{
The Sense of Ensemble: a Machine Learning Approach to Expressive Performance Modeling in String Quartets
}

\author{
Marco Marchini ${ }^{*}$, Rafael Ramirez ${ }^{*}$, Panos Papiotis ${ }^{*}$, Esteban Maestre* \\ *Music Technology Group, Universitat Pompeu Fabra, Spain \\ marco.marchini@upf.edu
}

\begin{abstract}
Computational approaches for modeling expressive music performance have produced systems that emulate music expression, but few steps have been taken in the domain of ensemble performance. In this paper, we propose a novel method for building computational models of ensemble expressive performance and show how this method can be applied for deriving new insights about collaboration among musicians. In order to address the problem of inter-dependence among musicians we propose the introduction of inter-voice contextual attributes. We evaluate the method on data extracted from multi-modal recordings of string quartet performances in two different conditions: solo and ensemble. We used machine-learning algorithms to produce computational models for predicting intensity, timing deviations, vibrato extent, and bowing speed of each note. As a result, the introduced inter-voice contextual attributes generally improved the prediction of the expressive parameters. Furthermore, results on attribute selection show that the models trained on ensemble recordings took more advantage of inter-voice contextual attributes than those trained on solo recordings.
\end{abstract}

\section{Introduction}

Music performance plays an important role in our culture. Research on expression in music performances (for an overview see (Gabrielsson, 1999, 2003)) investigates the manipulation of sound properties such as pitch, timing and amplitude in an attempt to understand and recreate expression in performances. In western music tradition, notation only partially specifies the manipulation of such sound properties, and it is often the performer who decides how to perform a piece. Musicians always introduce such deviations, even when playing mechanically (Palmer 1997).

Expressive music performance has been studied in the past from a computational perspective, which has produced expressive models capable of emulating human expression. 
Several machine learning techniques have been used as an approach to investigate expressive performance. Widmer et al. (Widmer \& Goebl 2004) and others have trained expressive models from real piano-performance data. A few other works have considered other instruments and genres (e.g. Mantaras et al. 2002 and Ramirez et al. 2006), which address additional expressive manipulations that are absent in piano performances (e.g., vibrato and glissando).

Computer systems of expressive music performance generally employ music analysis procedures in which score is translated into a sequence of feature vectors, each describing one note (Kirke \& Miranda, 2013). Each feature vector describes the nominal properties of a note as well as the score context in which the note appears (e.g. melodic/rhythmic contours and harmony). Computational models of expressive performance take feature vectors as input to produce predictions of expressive parameters (e.g. intensity and timing transformations). In data-driven systems, the computational models are built automatically by feeding machine learning algorithms with training examples. Training examples are collected by extracting note expressive parameters from recordings of humans' music performances along with feature vectors from the score. Such computational models can therefore be used to reproduce expressive performance of new untrained music scores by applying the predicted expressive parameters to (otherwise robotic) computer score renderings. Computational models are also used as a mean to investigate how humans play music expressively. This is possible either when the knowledge of the trained models is explicit and can thus be easily translated to human readable rules revealing recurrent performance patterns (Widmer 2002), or when models trained on different musicians and/or different performing conditions are compared (Poli 2004).

Despite the different applications of machine learning to expressive performance, most research papers focused on solo performances and did not address the problem of modeling ensemble expressive performance. In this paper we propose a method to deal with two main issues arising in ensemble music: polyphonic expression (each musician plays their melody with possibly a different expression respect to the one of the other concurrent voices) and interdependence among musicians (each musician takes into account information about concurrent voices to shape their expression). Firstly, to deal with polyphonic expression, we assign separate models of expression to each musician. This means that, as it happens in human performances, simultaneous voices might have different expressivity (e.g. the case where a voice containing the leading melody is played loud while the accompaniment voices are played soft). Secondly, to deal with inter-dependence among musicians, we extend the music analysis procedure in order to include information about other voices in the feature vectors. The models can thus take advantage of information about other voices (including score melodic/rhythmic information and also expressive parameters used by other) to predict outcome of expressive parameters.

The proposed approach has its roots in theoretical research of joint action in music performance. From a theoretical point of view, existing research (Keller, 2008) has pointed out three key cognitive processes present in ensemble musicians: auditory-imagery, where the musicians have their own anticipation of their own sound as well as the overall sound of the ensemble, prioritized integrative attention, where musicians divide their attention between their own actions and the actions of others, and adaptive timing, where each musician adjusts the performance to maintain temporal synchrony. This perspective makes it possible to identify the following important points: first, that each musician incorporates the ensemble score as well as 
the performance of the rest of the ensemble into an anticipation of the produced result. Second, the musician defines the saliency of each performed note with respect to the ensemble as a whole, shaping their performance so that it integrates both with the ensemble's actions as well as personal expressive choices. Lastly, the above choices must be made while maintaining ensemble synchrony at the same time.

Previous research on ensemble music performance focused mostly on synchronization aspects while neglecting the expressivity and the role of the music score. Some studies have focused on the synchronization on the task of tapping together (Repp 2005) or on very specific musical skills (Moore et al. 2010). Goebl \& Palmer (2009) studied the synchronization among musicians also taking into account the role and the auditory feedback.

To the best of our knowledge, to date only Sundberg et al. (1989) have addressed the issue of the ensemble expressive performance of string quartets. However, our paper differs from Sundberg et al.'s in that we adopt a data-driven approach based on recording ensemble performances while they take an analysis-by-synthesis approach. In other words, the models proposed by Sundberg et al. were manually defined and tested by creating synthetic performances (i.e. not by learning from humans' performances).

In this study we focused on expressive performance in string quartets classical music. We collected our performance data by recording a string quartet playing music excerpts from their repertoire and extracting the following note-level expressive parameters of the performance: sound level, bow velocity, note duration and vibrato extent. We recorded the string quartet performing with different expressive intentions (normal, mechanical, exaggerated) and under different performing conditions (each musician playing solo and in the ensemble). Regarding music analysis, we defined a set of contextual descriptors, extracted from the score, which describe the context in which each target note appears. The contextual descriptors describe the relative properties of neighboring notes and melodies respect to the target note. We say that a contextual descriptor is horizontal if it describes notes/melodies belonging to the same voice as the target note, and we say that it is vertical if it describes notes/melodies belonging to the other voices.

We trained separate models of expressions for each musician with machine learning algorithms, which perform regressions over the considered expressive parameters. We include in the feature vector of the models the contextual descriptors starting from a feature set of only horizontal descriptors to which we add vertical descriptors in two steps. We then expand the complete descriptors set in two further steps, by including, first, the recent performed expressive parameters on the note previous to the target note, and second, the concurrent performed expressive parameters by other musicians.

The purpose of training expressive performance models on each musician separately and introducing inter-voice dependencies is two-fold. First, this work can be viewed as a proof-ofconcept application of expressive performance modeling in music ensembles. And second, by comparing models obtained on different expressive parameters, different musicians and different playing condition we investigate on several aspects of ensemble performance. Rather than presenting final answers to broad ensemble performance aspects, which might require a considerably larger amount of data, we discuss results on specific aspects, which highlight the potentials of using this method to study ensemble expressive performance. 
In particular, difference between playing solo and in ensemble was considered both as a sanity-check test - to see whether the models make sense of the data - and as mean to understand in which part of the trained models should we look for signs of inter-dependence. Additionally, we look for differences between the considered expressive parameters to understand which of them was shaped as a result of inter-dependence among ensemble voices, and which only a product of the individual voices.

The rest of the paper is structured as follows. Section 2 examines the acquisition of the data, the analysis of the performance and the definition of score contextual descriptors. Section 3 describes the methodology used for expressive performance modeling including the machinelearning algorithms and evaluation. We, then, present and interpret the results in Section 4. And provide our conclusions in Section 5.

\section{Score and Performance Analysis}

\subsection{Music Material}

The material studied in this paper was recorded by a professional string quartet. The subjects were members of a professional string quartet that had already been performing together for more than a year. The mean age of the musicians was 30 years old, and all had at least 10 years of experience as professional musicians. To ensure that the ensemble was familiar with the material and that the performers shared a common understanding of the underlying score, we asked the performers to give us their current repertoire as well as propose which pieces they were most comfortable with performing in a concert scenario.

Two different experimental scenarios were used. In the first scenario, we asked the quartet to perform an entire piece with three different types of expressive intentions (mechanical, normal, exaggerated). In the second scenario, we asked the quartet to perform short excerpts from their repertoire under two conditions (ensemble and solo).

\subsubsection{Expressive Intentions scenario}

We selected the longest (and most recently performed) piece in the quartet's current repertoire, Beethoven's String Quartet No. 4 (Opus 18) 4th movement (bars 584-714). The musicians were instructed to perform the entire piece three times, once for each of the following expressive intentions:

- Mechanical, where the musicians stayed as 'faithful' to the score as possible without introducing expressive deviations.

- Normal, where the musicians performed the piece as they would in a concert scenario.

- Exaggerated, where the musicians introduced 'extreme' expressive deviations from the score, in comparison to the 'normal' condition.

The instructions regarding the performers' expressive intent have been shown to be clearly understood by musicians and non-musicians alike (Kendall and Carterette, 1990). The purpose of the above scenario was to capture different degrees of 'deviating from the score', i.e., introducing personal choices which are not explicitly stated. Moreover, the Mechanical and 
Exaggerated conditions provided two 'extreme' cases of ensemble performance, which are plausible performance conditions per se, while at the same time requiring different types of collaboration and communication from the musicians.

In total, the three repetitions consisted of 7508 notes (2520 notes for violin 1, 1889 for violin 2, 1683 for viola and 1416 for cello).

\subsubsection{Solo vs. Ensemble scenario}

For the second scenario, we selected short excerpts from the quartet's repertoire: four excerpts from the same Beethoven's String Quartet No. 4 (Opus 18). The selected excerpts correspond to bars 54-78, 138-151, 491-534 and 602-619 respectively. These short excerpts were then recorded under the following two conditions:

- Solo, where the musicians each performed their own voice alone (without listening to pre-recorded material or a metronome signal).

- Ensemble, where the entire quartet performed together following a brief rehearsal.

The purpose of the above scenario was to observe whether the musicians would perform their voices differently from one condition to the other, given that in the solo condition there were no external perturbations to the performance, nor any external feedback for the musicians' actions. The solo and ensemble recordings were made on different days.

In total, the four excerpts comprised of 2068 notes (440 notes for violin 1, 535 for violin 2, 549 for viola and 544 for cello).

\subsection{Audio and Motion Data Acquisition}

The acquired data include four individual audio tracks (one for each musician, all sampled at $44100 \mathrm{~Hz}$ ) from piezoelectric bridge pickups (models: Fishman V-100 and Fishman C-200) installed on the instruments. Pickup gains were manually set with the aid of level meters in the recording equipment to avoid clipping of individual audios. To balance the sound level of the quartet as a whole, we employed an iterative procedure, based on listening to the mix of the pickups on the headphones while adjusting the four gains. We also synchronously acquired bowing motion data at a sample rate of $240 \mathrm{~Hz}$ by means of a Polhemus Liberty wired motion capture system (based on electromagnetic field sensing) as detailed in (Maestre 2009). In a post-acquisition step, the synchronization of audio and motion capture data were reviewed through the alignment of linear timecode timestamps.

\subsection{Pre-processing of Audio and Motion Data}

Audio data pre-processing included the extraction of sound level, fundamental frequency, and aperiodicity. Sound level was computed in $\mathrm{dB}$ from the root mean square of the samples contained in overlapping windows while fundamental frequency and aperiodicity were obtained via an autocorrelation-based F0 estimation method as described in (de Cheveigne \& Kawahara, 2002). 
Motion data pre-processing involved extracting time series features with estimations of bow transversal velocity and bow pressing force, following the methods detailed in (Maestre, 2009) and (Marchini et al., 2011).

The time-series of audio and motion capture features of each musician were used together with a symbolic representation of the score to automatically obtain precise note-by-note segmentations by means of a score-performance alignment algorithm (Maestre 2009) through dynamic programming. The automatically estimated note boundaries were manually inspected to correct possible segmentation errors.

\subsection{Performance Analysis}

Once audio and motion data were synchronized and score-performance alignments were carried out through note-level segmentation, we extracted the following performance parameter descriptors from each note in the recordings: sound level, note lengthening, vibrato extent, and bow velocity.

\section{Sound level}

Sound level was estimated for each note as follows. First, the audio signal corresponding to each voice was divided into frames of $23 \mathrm{~ms}$ with an $82 \%$ overlap. Let $\left(x_{1}^{(i)}, x_{n}^{(i)}\right)$ be the samples of the $i$-th frame. The energy of the frame was computed as the root mean square of its samples:

$$
\operatorname{RMS}(i)=\frac{1}{n} \sqrt{\left(x_{1}^{(i)}\right)^{2}+\cdots+\left(x_{n}^{(i)}\right)^{2}}
$$

We then obtained the sound level $\mathrm{SL}(i)$ by expressing the RMS energy in $\mathrm{dB}$ :

$$
\operatorname{SL}(i)=20 \log _{10}(\operatorname{RMS}(i))
$$

Lastly, the note sound level attribute for each note $N_{k}$ was computed as the maximum sound level value SL(i) within the note boundaries

$$
\mathrm{NSL}_{k}=\max _{i \text { in } \mathrm{N}_{\mathrm{k}}} \operatorname{SL}(i)
$$

\section{Note lengthening}

The score dictates note durations in beats. Since the duration of a beat changed during the course of a performance due to ensemble tempo modulations, here we consider note lengthening as being relative to the local tempo of the ensemble. We define note lengthening as the ratio between the nominal duration of a note (defined by the local tempo of the ensemble) and the actual duration of each performed note. We chose to consider duration as being such in an attempt to remove the effect of broad tempo modulations and focus only on residual timing deviations, as it is often done in studies of expressive performance (Widmer \& Tubodic 2003).

A necessary pre-processing step is the estimation of the ensemble tempo for each note in a local context, thereby making it possible to express a nominal duration in seconds. A first approach to estimating the tempo would be to divide the total number of beats recorded by the total performance duration in seconds to derive an average tempo. However, this approach totally neglects possible changes of tempo, which might occur during the performance. For this 
reason we divided the piece into phrases ${ }^{1}$ with the help of a professional musicologist and looked for possible changes of tempo. By looking at the distributions of note durations in the performance it became evident that abrupt changes of tempo appear at section changes. On the other hand, tempo stays nearly constant within sections. For these reasons, we chose the average tempo of the section as the reference tempo for each note. The note lengthening is computed with the formula:

$$
\mathrm{NL}_{k}=\frac{\text { notePerformedDuration }}{\text { noteScoreDuration }} \times \text { sectionBPS }
$$

where notePerformedDuration is the performed duration in seconds, noteScoreDuration is the nominal duration of the note in beat units and sectionBPS is the average beats per second of the section where the note is played. Where the note was followed by a pause we included the duration of the pause in the note enabling us to use the inter onset interval (IOI) to estimate notePerformedDuration.

It is worth mentioning that in the case of ensemble recordings we used the same sectionBPS of the four musicians. On the other hand, for the solo recordings we use a different value of sectionBPS for each musician since they each employed a slightly different tempo.

\section{Vibrato extent}

Vibrato is an expressive manipulation of pitch corresponding to a frequency modulation of F0 (fundamental frequency) characterized by its rate and extent (Prame, 1997). We considered only the extent of the vibrato since the rate is only defined for notes with vibrato and thus plays a secondary role on expression. However, the procedure we implemented to extract the vibrato estimates both rate and extent at the same time.

We estimated the vibrato of each note from a filtered F0 time series within the note boundaries. The procedure is based on finding the amplitude of the sinusoid that correlates best to the filtered FO trajectory. Such amplitude is used as the estimated vibrato extent of the note. Modulations of vibrato extent within the note boundaries were thus implicitly discarded favoring the selection of the highest vibrato extent reached within the note.

The first step was to filter from the F0 trajectory the transitions from one note to the next characterized by low aperiodicity. Suppose that note $k$ has $n_{k}$ frames within its onset and offset. Each of such frames $i=1, \ldots, n_{k}$ is described by the triplet $\left(t_{i}^{(k)}, f_{i}^{(k)}, a_{i}^{(k)}\right)$ with, respectively, the frame time, the fundamental frequency (F0) and the aperiodicity as obtained with the autocorrelation-based F0 estimation algorithm (de Cheveigne \& Kawahara, 2002). We use the threshold $A$ to assign weights $w_{i}^{(k)}$ to each frame in the following manner:

$$
w_{i}^{(k)}=\left\{\begin{array}{ll}
1 & \text { if } a_{i}^{(k)} \leq A \\
0 & \text { if } a_{i}^{(k)}>A
\end{array} \quad i=1, \ldots, n_{k}\right.
$$

We then computed for each note the weight center of mass (in time) $\mu^{(k)}$ and the standard deviation $\sigma^{(k)}$ for each note using the formulae:

\footnotetext{
${ }^{1}$ This procedure led to 128 phrases from the four excerpts jointly and 53 phrases for each of the expressive intention piece. The phrases were hierarchically linked so as to merge in higher time spans, up to whole sections of the piece.
} 


$$
W^{(k)}=\sum_{i=1}^{n_{k}} w_{i}^{(k)}, \quad \mu^{(k)}=\frac{1}{W^{(k)}} \sum_{i=1}^{n_{k}} w_{i}^{(k)} t_{i}^{(k)}, \quad \sigma^{(k)}=\sqrt{\frac{1}{W^{(k)}} \sum_{i=1}^{n_{k}} w_{i}^{(k)}\left(t_{i}^{(k)}-\mu^{(k)}\right)^{2}}
$$

The select part of note $k$ is then obtained by removing all the frames $i$ such that

$$
\left|t_{i}^{(k)}-\mu^{(k)}\right| \geq B \sigma^{(k)}
$$

where $B$ is an opportune positive threshold.

Comparing the plots of $\mathrm{FO}$ and aperiodicity we noticed that the $\mathrm{F} 0$ is generally reliable when the aperiodicity is lower than $A=0.2$. We then tested several values for $B$ and selected $B=1.8$ as a good compromise between discarding too much data and getting the most reliable part of the F0. This value is such that most times the whole note is selected, except for very long sustained notes where some boundaries at the end are discarded.

Once the F0 time series has been filtered we proceeded to estimate the vibrato extent on the selected part of F0. We employed frequency-domain analysis following a similar procedure as the one described in (Herrera, 1998). We performed a Fourier analysis of the filtered F0 and looked for peaks in the spectrum ranging between 4 and $8 \mathrm{~Hz}$. If one or more peaks were found in this range we select the one corresponding to the sinusoidal component with the highest amplitude. The amplitude of such sinusoid (in pitch cents) is our estimation of vibrato extent whereas its frequency is the vibrato rate. Where no salient peak was detected or the select part of F0 was shorter than every possible vibrato period (125 ms), the vibrato extent was set at zero.

\section{Bow velocity}

Bow transversal velocity is one of the main control parameters available for the string player to convey expression, affecting timbre and loudness (Askenfelt, 1986). We measured bow transversal velocity from each frame of motion capture data as described in (Maestre, 2009), leading to a bow velocity time series expressed in $\mathrm{cm} / \mathrm{s}$ and sampled at $240 \mathrm{~Hz}$. Bow transversal velocity can be positive (down-bow) or negative (up-bow), but since we were interested in the speed of bowing we first computed the absolute value of bow velocity samples

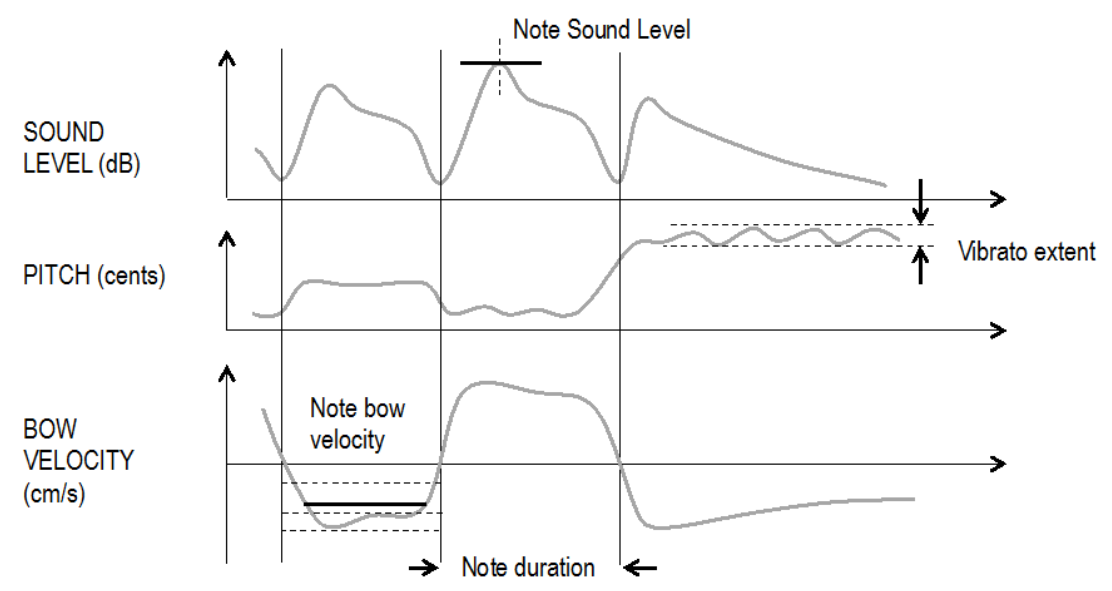

Figure 1. An example of the Sound Level, Pitch and Bow Velocity time series along with the descriptors extracted at the note level. 
across each note. Then, in order to extract a representative bow velocity value for each note, we computed the interquartile mean on the absolute values of each note segment.

Figure 1 shows an example of the time series that are extracted from the raw data as well as the descriptors computed for each note segment.

\subsection{Score Descriptors}

One of the main goals of research on expressive performance is relating score contextual information to expressive parameters. In ensemble scores we have more than one concurrent voice being played simultaneously. Each of those voices can be individually analyzed to extract melodic/rhythmic context related to a reference note. However, the same melodic line can assume different connotations if the accompaniment is altered and, in this case, the musician

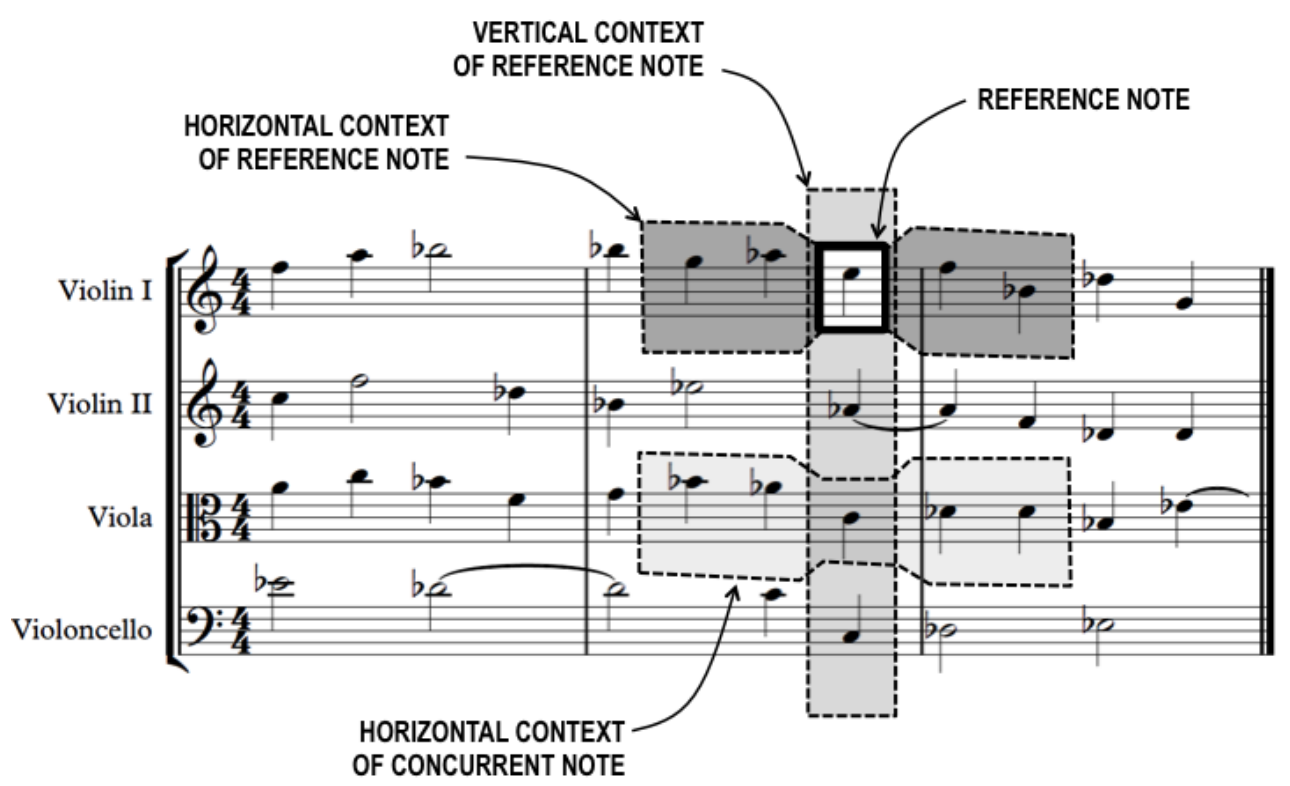

Figure 2. An example demonstrating the Horizontal and Vertical contexts as they are derived from the score

might decide to render it employing different expressivity. For this reason, contextual information that only describes one melodic line is not sufficient for predicting the performance. In this case we deal with four voices, which is a good compromise between a duet and an orchestra; there being enough inter-voice relations and contextual information to make a specific analysis meaningful, but not too many to dramatically increase the complexity and make the analysis impossible.

We introduced a set of ensemble context descriptors, which can be extracted, in theory, from any ensemble score. Figure 2 graphically depicts the kind of score contextual descriptors we considered on a real score example. Horizontal context descriptors were computed based solely on a musician's individual voice, ignoring the voices of the others (see Table 1). These include both properties of the note itself, as well as properties of the neighboring notes (preceding and subsequent) in that voice. Different temporal context windows sizes were considered by adding 
more or less neighboring notes to the feature set. Vertical context descriptors include information from the score about the notes played by other musicians concurrently with the reference note (see Table 2). In each of the three other voices, the corresponding concurrent note event was selected as the one note (or rest) that is active (or starts) at the beat position where the reference note starts. Vertical descriptors were then computed on the selected concurrent notes by either relating their properties to the reference, or combining their properties to compute resuming descriptors. The horizontal context of each concurrent note event was also considered as a vertical context of the reference note (see Table 3).

In Tables 1-3 we list all of the considered score contextual descriptors in detail. We assume that the reference note $N_{0}$ has its onset a beat $b_{0}$ in the score, has pitch $p_{0}$, and duration $d_{0}$. At beat $b_{0}$ the other musicians are playing notes $N_{0}^{(1)}, N_{0}^{(2)}$ and $N_{0}^{(3)}$ respectively. We analogously use superscripts to refer to properties of such notes; e.g., $p_{0}^{(2)}$ is the pitch value of the second of

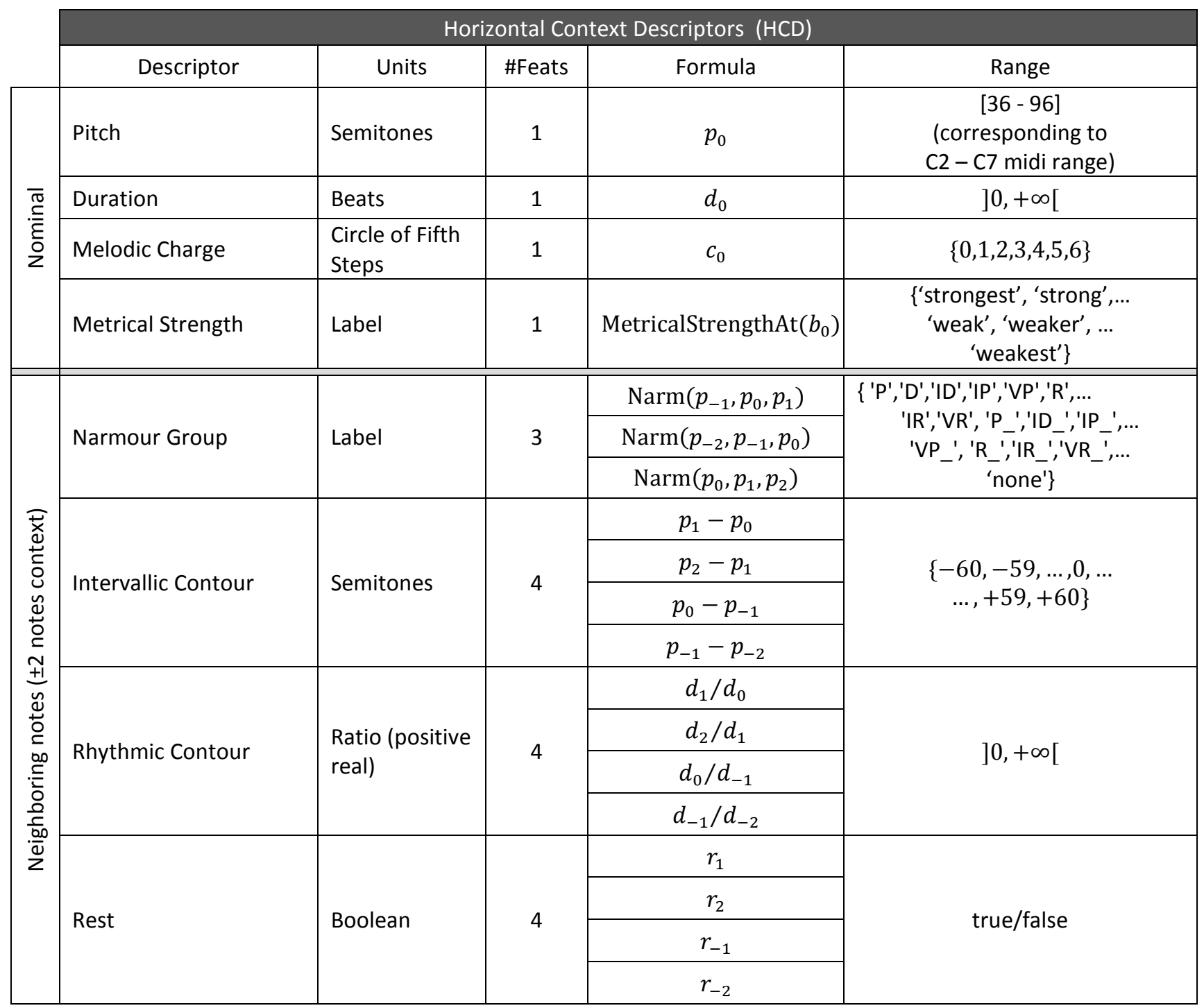

Table 1. Horizontal context descriptors. 


\begin{tabular}{|c|c|c|c|c|}
\hline \multicolumn{5}{|c|}{ Vertical Context Descriptors (VCD) } \\
\hline icriptor & Units & \#Feats & Formula & \\
\hline & \multirow{3}{*}{ Boolean } & \multirow{3}{*}{3} & $r_{0}^{(1)}$ & \\
\hline & & & $r_{0}^{(2)}$ & \\
\hline & & & $r_{0}^{(3)}$ & \\
\hline \multirow{3}{*}{ : Interval } & \multirow{3}{*}{ Beats } & \multirow{3}{*}{3} & $b_{0}^{(1)}-b_{0}$ & \\
\hline & & & $b_{0}^{(2)}-b_{0}$ & \\
\hline & & & $b_{0}^{(3)}-b_{0}$ & \\
\hline \multirow{3}{*}{ itch } & \multirow{3}{*}{ Semitones } & \multirow{3}{*}{3} & $p_{0}^{(1)}-p_{0}$ & \multirow{3}{*}{$\begin{array}{r}\{-61 \\
\ldots\end{array}$} \\
\hline & & & $p_{0}^{(2)}-p_{0}$ & \\
\hline & & & $p_{0}^{(3)}-p_{0}$ & \\
\hline \multirow{3}{*}{ Iuration } & \multirow{3}{*}{$\begin{array}{l}\text { Ratio } \\
\text { (positive } \\
\text { real) }\end{array}$} & \multirow{3}{*}{3} & $d_{0}^{(1)} / d_{0}$ & \\
\hline & & & $d_{0}^{(2)} / d_{0}$ & \\
\hline & & & $d_{0}^{(3)} / d_{0}$ & \\
\hline \multirow{2}{*}{ Charge } & \multirow{2}{*}{$\begin{array}{l}\text { Real, and a } \\
\text { Boolean }\end{array}$} & \multirow{2}{*}{2} & HarmonicChargeAt $\left(b_{0}\right)$ & \\
\hline & & & $c_{0} \geq \max \left(c_{0}^{(1)}, c_{0}^{(2)}, c_{0}^{(3)}\right)$ & \\
\hline Inter-Beat & Beats & 1 & $\max \left(b_{0}^{(1)}, b_{0}^{(2)}, b_{0}^{(3)}\right)-b_{0}$ & \\
\hline Relative & Ratio & 1 & $\min \left(d_{0}^{(1)} / d_{0}, d_{0}^{(2)} / d_{0}, d_{0}^{(3)} / d_{0}\right)$ & \\
\hline I Relative & Ratio & 1 & $\max \left(d_{0}^{(1)} / d_{0}, d_{0}^{(2)} / d_{0}, d_{0}^{(3)} / d_{0}\right)$ & \\
\hline $\begin{array}{l}\text { Relative } \\
\text { harge } \\
\text { l }\end{array}$ & $\begin{array}{l}\text { Difference } \\
\text { (integer) }\end{array}$ & 1 & $\min \left(c_{0}^{(1)}-c_{0}, c_{0}^{(2)}-c_{0}, c_{0}^{(3)}-c_{0}\right)$ & -1 \\
\hline $\begin{array}{l}\text { I Relative } \\
\text { harge } \\
\text { ) }\end{array}$ & $\begin{array}{l}\text { Difference } \\
\text { (integer) }\end{array}$ & 1 & $\max \left(c_{0}^{(1)}-c_{0}, c_{0}^{(2)}-c_{0}, c_{0}^{(3)}-c_{0}\right)$ & \\
\hline
\end{tabular}

Tahle 2 Vertiral rontext decrintorc

the other musicians. We use the subscripts to indicate neighboring notes; e.g. $d_{-1}$ and $d_{1}$ refer, respectively to the duration of the previous and the following note in the voice line of $N_{0}$. We combine both subscripts and superscripts to refer to neighboring notes in the voice of other musicians; e.g., $p_{-1}^{(1)}$ is the pitch of $N_{-1}^{(1)}$, which is the note previous note to the concurrent one, in the voice line of the first of the other musicians. In the following sections we explain how melodic, rhythmic and harmonic descriptors are defined and extracted from the score.

\subsubsection{Melodic Descriptors}

We include the nominal pitch of the reference note and a set of melodic descriptors to characterize the melodic line of the target voice. The Intervallic contour (see Table 1) is represented by a collection of melodic intervals (signed difference of pitch semitones) for each couple of consecutive notes. 


\begin{tabular}{|c|c|c|c|c|}
\hline \multicolumn{5}{|c|}{ Score Context of Other Voices (SCOV) } \\
\hline iptor & Units & \#Feats & Formula & Ral \\
\hline \multirow{6}{*}{$=$} & \multirow{6}{*}{ Semitones } & \multirow{6}{*}{6} & $p_{1}^{(1)}-p_{0}^{(1)}$ & \multirow{6}{*}{$\begin{array}{r}\{-60,-5 \\
\ldots,+5\end{array}$} \\
\hline & & & $p_{0}^{(1)}-p_{-1}^{(1)}$ & \\
\hline & & & $p_{1}^{(2)}-p_{0}^{(2)}$ & \\
\hline & & & $p_{0}^{(2)}-p_{-1}^{(2)}$ & \\
\hline & & & $p_{1}^{(3)}-p_{0}^{(3)}$ & \\
\hline & & & $p_{0}^{(3)}-p_{-1}^{(3)}$ & \\
\hline \multirow{6}{*}{ : Contour } & \multirow{6}{*}{$\begin{array}{l}\text { Ratio } \\
\text { (positive } \\
\text { real) }\end{array}$} & \multirow{6}{*}{6} & $d_{1}^{(1)} / d_{0}^{(1)}$ & \multirow{6}{*}{ ]0, } \\
\hline & & & $d_{0}^{(1)} / d_{-1}^{(1)}$ & \\
\hline & & & $d_{1}^{(2)} / d_{0}^{(2)}$ & \\
\hline & & & $d_{0}^{(2)} / d_{-1}^{(2)}$ & \\
\hline & & & $d_{1}^{(3)} / d_{0}^{(3)}$ & \\
\hline & & & $d_{0}^{(3)} / d_{-1}^{(3)}$ & \\
\hline \multirow{9}{*}{ Group } & \multirow{3}{*}{ Label } & \multirow{3}{*}{3} & $\operatorname{Narm}\left(p_{-1}^{(1)}, p_{0}^{(1)}, p_{1}^{(1)}\right)$ & \multirow{3}{*}{$\begin{array}{l}\{\text { 'P',''D','ID',' } \\
\text { 'IR','VR', 'P_' } \\
\text { 'VP_', 'R__', } \\
\text { 'no }\end{array}$} \\
\hline & & & $\operatorname{Narm}\left(p_{-1}^{(2)}, p_{0}^{(2)}, p_{1}^{(2)}\right)$ & \\
\hline & & & $\operatorname{Narm}\left(p_{-1}^{(3)}, p_{0}^{(3)}, p_{1}^{(3)}\right)$ & \\
\hline & \multirow{6}{*}{ Boolean } & \multirow{6}{*}{6} & $r_{1}^{(1)}$ & \multirow{6}{*}{ true, } \\
\hline & & & $r_{-1}^{(1)}$ & \\
\hline & & & $r_{1}^{(2)}$ & \\
\hline & & & $r_{-1}^{(2)}$ & \\
\hline & & & $r_{1}^{(3)}$ & \\
\hline & & & $r_{-1}^{(3)}$ & \\
\hline
\end{tabular}

Table 2. Score context of other voices.

Furthermore, we implement the Narmour implication realization model in each group of three consecutive notes (Narmour 1990). The Narmour group $\operatorname{Narm}(x, y, z)$ of three consecutive pitches $x, y$ and $z$ describes how the expectation that is built over the sequence of pitches $(x, y)$ is fulfilled by the pitch $z$. We encoded this as class labels; of which there are 15 possible labels (see Table 1) to which we add an additional 'none' label for the case that one of the three notes is a rest.

The melodic charge $c$ is a descriptor of note salience, which is defined as the smallest number of steps required to reach the note in the circle of fifths from the tonic note (a number from zero to six). The melodic charge was considered a horizontal descriptor since it can be computed independently from the concurrent melodic voices by knowing the tonality alone.

\subsubsection{Rhythmic Descriptors}

Rhythmic information of the target voice was included by describing the durations of 
neighboring notes, relations to the meter and eventual pauses. We employed the metrical strength and rhythmic contour as well as a Boolean variable indicating the presence of neighboring rests (see Table 1 ).

Metrical strength depends on the position of the note relative to the bar meter and is encoded by a class label from strongest to weakest depending on the beat position $b_{0}$ of the reference note. For the 4:4 meter we define the metrical strength, per case, as follows, where the first case that matches apply:

$$
\text { MetricalStrengthAt }\left(b_{0}\right)=\left\{\begin{array}{c}
\text { 'strongest', if } b_{0} \text { is at the } 1 \text { st beat of the bar } \\
\text { 'strong', if } b_{0} \text { is at the up-beat ( } 3 \mathrm{rd} \text { beat of the bar) } \\
\text { 'weak', if } b_{0} \text { is at a quarter (non mentioned above) division of the bar } \\
\text { 'weaker', if } b_{0} \text { is a eighth (non mentioned above) division of the bar } \\
\text { 'weakest', in all other cases }
\end{array}\right.
$$

The pieces in our recordings have the following meters: $2: 2,4: 4$ and 3:4. We treated 2:2 and $4: 4$ in exactly the same way. In the case of the ternary meter we applied the same definition as 4:4 except that there are two up-beats in the $2^{\text {nd }}$ and the $3^{\text {rd }}$ positions.

Rhythmic contour was characterized by the ratios between consecutive nominal durations of neighboring notes.

We also introduced rhythmic information derived from the other voices of the ensemble (see Table 2). For each concurrent note, we computed the relative duration with respect to the reference note (ratio of nominal durations). The inter-beat interval (IBI) is the difference of start times between the reference note and the concurrent note (in beats). Additionally, we computed maximum and minimum values across all the concurrent relative durations and the minimum IBI.

\subsubsection{Harmonic Descriptors}

Harmonic relations between voices are very important in ensemble performances, especially where string instruments are concerned given that intonation is not fixed to specific intervals but rather continuous. We computed the harmonic charge (Friberg 1995) on all the notes active within the beat $b_{0}$ of the reference note. To compute the harmonic charge, the pitches of notes active within the beat are preliminarily collected in a list. By using the implementation from the open project music $21^{2}$ we constructed a chord with the list of pitches and then estimated its root note. We then computed the melodic charge of each note in the chord with respect to the root note. Lastly, we calculated the mean of the obtained melodic charges resulting in the value of harmonic charge HarmonicChargeAt $\left(b_{0}\right)$.

Other harmonic descriptors were provided by the relative melodic charge. We computed the difference in melodic charge of every concurrent note with the reference note. We also stored the minimum and the maximum value of those differences (minRMC and maxRMC, see Table 2). This last descriptor also describes the salience of the note in the vertical context; a similar descriptor was used by Sundberg et. al 1989 where the reference note is defined as the most salient note if maxRMC is less than/equal to zero.

\footnotetext{
${ }^{2}$ http://mit.edu/music21/
} 


\section{Expressive Performance Modeling}

\subsection{Learning task}

Data-driven performance models predict note-level expressive transformations given a set of contextual descriptors of the note. Here, we are interested in learning a function $f$ of the form

$$
f(\text { Note }) \rightarrow(S L, B V, V E, N L)
$$

where Note is a note characterized by the set of descriptors described in Section 2.5, and SL, $\mathrm{BV}, \mathrm{VE}$, and $\mathrm{NL}$ are, respectively, the predicted sound level, bow velocity, vibrato extent and note lengthening. Each expressive manipulation of the note is here a different learning task on which we perform a regression.

\subsection{Learning Algorithms}

The machine learning techniques considered in this paper are the following:

- Model Trees. A decision tree classifier recursively constructs a tree by selecting the most relevant attribute at each node. This process gradually splits up the training set into subsets until all instances at a node have the same classification. The selection of the most relevant attribute at each node is based on the information gain associated with each node of the tree (and corresponding set of instances). A model tree, instead of predicting a class, takes into account all instances in a leaf of the decision tree and generates a linear regression in order to compute a real value as prediction. We have applied the model trees rule algorithm implemented in the Weka data mining software (Hall et al, 2009).

- Support Vector Machines (SVM). SVM (Cristianini, 2000) take great advantage of using a non-linear attribute mapping which enables them to be able to predict non-linear models (though they remain linear in a higher dimension space). Thus, they provide a flexible prediction, but with a higher computational cost necessary to perform all the calculations in a higher dimensional space. The classification accuracy of SVM largely depends on the choice of the kernel evaluation function and the parameters which control the amount to which deviations are tolerated (denoted by epsilon). In this paper we have used SVM with a linear kernel.

- $K$-Nearest Neighbor (kNN). This is one of the simplest non-parametric machine-learning algorithms. It predicts the value of a test instance by assigning to it an average value of the $\mathrm{k}$ closest instances in the training set. The distance between instances is based on the Euclidian distance in the feature space. In this article we use it with $k=1$ as a baseline approach. 
We evaluated the above algorithms by means of a cross-validation procedure obtaining an average value of correlation coefficient. The folds were created using phrases of around 16 beats (as provided by the musicological analysis) in order to avoid phrases spanning both test and training set. In order to further guarantee the validity of the results, when creating each fold we removed all repetitions of notes, due to repeated sections (notated with and/or without repeat signs), in the test set from the training set. Doing so left 27 folds for each of the expressive intention corpus and 25 folds for each of the excerpt corpus.

We used the correlation coefficient to measure how well the algorithm was capable of generating a meaningful prediction.

\subsection{Feature Sets}

In a preliminary study (Marchini et al, 2013) we tested different combinations of score contextual features considering, among other aspects, the size of the temporal window. We considered a range of window sizes spanning from one to five neighboring notes. This was repeated either for a set of horizontal features or a set of horizontal+vertical features. We found no evidence that larger window sizes improved the prediction. For this reason we limited this extended study to a fixed context length of two neighboring notes. The introduced restriction allowed us to focus mainly on the type of attribute by limiting the number of attributes to a reasonable number.

We selected five different feature sets (FS) containing an increasing number of contextual attributes of different types. In the first three feature sets we start with information of one's individual voice and progressively add contextual information about vertical context:

FS1) Horizontal score context of the target voice (Table 1)

FS2) FS1 + vertical score context of concurrent notes (Table 2)

FS3) FS2 + horizontal context of concurrent notes (Table 3)

In addition to score context we also incorporate the expressive parameter values employed in the two previous notes in this performance of the score. We include first the expressive parameters of the target musician, and then add concurrent expressive parameters of the other musicians:

FS4) FS3 + values of expressive parameter values of the two previous notes in the target voice

FS5) FS4 + value of expressive parameter values of the concurrent notes and one note previous

The defined feature sets contain respectively 19, 38, 59, 61 and 67 attributes.

\subsection{Feature Selection}

The number of attributes is reduced when training the system by running a feature selection algorithm. After building each fold following the procedure explained in Section 3.2, we 
randomly split the training set into two halves. In the first half, we perform a feature selection algorithm in order to reduce the amount of attributes. We then train the regression of expressive parameter on the second half of instances using the attributes selected in the first half. Lastly, we used the regression to compute the predictions on the test set.

The employed feature selection algorithm attempts to find a subset of features $f_{1}, \ldots, f_{k}$ maximizing the Correlation Feature Selection (CFS):

$$
\frac{r_{c f 1}+r_{c f 2}+\cdots+r_{c f k}}{\sqrt{k+2\left(r_{f 1 f 2}+\cdots+r_{f i f j}+\cdots+r_{f k f 1}\right)}}
$$

where each $r_{c f i}$ is the correlation of each feature with the predicted attribute and $r_{f i f j}$ are the correlations between features (Hall, 1998). The idea behind CFS is that a good set of features is made out of attributes poorly correlated with each other while highly correlated with the predicted variable.

In order to find a set of features maximizing the CFS in a reasonable amount of time we reduced the number of combinations to test by employing a greedy step search. The algorithm starts with an empty set and adds one feature at a time. The feature that maximizes the CFS is selected at each step. It stops adding features when no further improvement of CFS is possible.

\subsection{Building the Datasets}

Before applying any machine-learning algorithm we needed to construct our dataset. The dataset is a collection of note instances, each characterized by a set of descriptor attributes and a target value. We created each dataset by choosing a musician, a corpus and a feature set (FS) to which one of the four corresponding expressive parameters was assigned as target value.

From the recorded excerpts and pieces we create 5 corpora:

- Normal Piece

- Mechanical Piece

- Exaggerated Piece

- Ensemble Excerpts, where all the note instances of the four excerpts played in ensemble are merged in one dataset

- Solo Excerpts, where all the note instances of the four excerpts played solo are merged in one dataset

For each of the five corpora we create 80 individual datasets with all the combinations given by:

- learning tasks: sound level / bow velocity / vibrato extent / note lengthening

- $\quad$ feature sets: FS1 / FS2 / FS3 / FS4 / FS5

- $\quad$ target musicians: violin 1 ( $\mathrm{vl} 1$ ) / violin 2 (vl2) / viola (vla) / cello (cello)

Two datasets having the same corpus, FS and musician, also share the same score contextual descriptor attributes. Indeed, score contextual attributes do not depend on the target value. The expressive parameters features in FS4 and FS5 are, instead, conventionally drawn from the same type of expressive parameter as the one of the target. For example, where 
vibrato extent was the target expressive parameter then the past performance parameter values were drawn from previous note vibrato extent values, whereas if sound level was the target expressive parameter then the past performance parameter values were drawn from previous note sound level values. It is worth noticing that in the case of the solo excerpts, the concurrent expressive performance values included in the feature set FS5 were not heard by the performers. However, we take it from the other musicians' solo performance when building FS5.

Each dataset was then used for executing one test using the previously described cross fold validation per phrase resulting in 400 tests each of which yielded a correlation coefficient value obtained using model trees with feature selection, removing repetitions from the test set and using cross-fold evaluation per phrase. We used the correlation coefficient as an estimation of the predictive power of the model. In Sections 4.1-4.2 we comment on how this correlation coefficient depends on different factors.

Additionally, we tested another two machine-learning algorithms (SVR and kNN), another type of validation (10-fold cross validation), the effect of feature selection and removing repetition. The end result of which is $3 \times 2 \times 2 \times 2 \times 400=9600$ individual tests. We discuss how these additional factors affect the correlation coefficient in Section 4.3.

\section{Results}

We computed correlation coefficients (using cross-validation) by running the machine learning algorithms once for each feature set (FS), each corpus, each musician and each learning task. At stage one we compared the performance of each of the collected setups by looking at how each factor considered affects it. In Figure 3 we show an example of the collected predictions along with the performed value of the four instruments.

As already mentioned in Section 3.5, we use the correlation coefficient as an estimation of the predictive power of the method. The sample correlation coefficient however is not normally distributed since its variance becomes smaller for higher values of correlation. We employed the Fisher z-transformation to correct each correlation coefficient $r$, which distributes the variance equally across all levels of correlation. The Fisher transformation is given by the following formula:

$$
z=\frac{1}{2} \ln \left(\frac{1+r}{1-r}\right)
$$




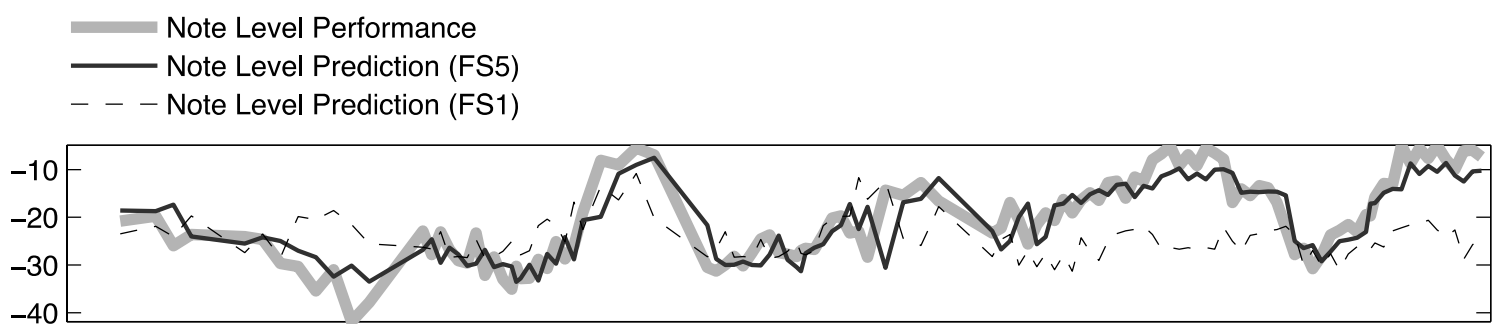

Figure 3. We represent the note sound level of the four musicians along with the predicted value (using model trees with the FS1 and FS5). The example is taken from Beethoven's String Quartet No. 4 (Opus 18) 4th movement. We show the exaggerated expressive execution.

We performed an Analysis of Variance (ANOVA with interaction) of the $z$ coefficients (after applying the Fisher z-transform in order to distribute the variance equally across all levels of correlations) obtained from all the considered factors (musician, FS, learning task and corpus). The learning task was responsible for the largest variance encountered. This means that on average some expressive parameters are easier to predict than others. Taking into account the variance, the learning task is followed by the feature set, the dataset and the musician, in that order. For these reasons, the results are more intelligible when each dataset is presented by feature sets, learning tasks and musician.

We present our results with model trees for the expressive intentions dataset (Section 4.1) and then for the performing conditions dataset (Section 4.2). We make some observations about how learning task, musician and feature set affect the result.

Furthermore, we show that our observations are consistent with other regression classifiers (SVR and kNN) and how other corrections we introduced in the evaluation procedure (removing repetitions and using cross fold validation per phrase) affected the result (see Section 4.3). 
Lastly, we show that the feature selection procedure, despite removing some of the attributes from the training set, does not worsen the prediction with respect to training with the full set of attributes. Hence, we report that the feature selection procedure tends to select an appropriate subset of features for each of the setups. We, then, present the results of feature selection on the excerpts datasets (without cross-validation) using the largest feature set FS5 for the solo vs. ensemble scenario. We demonstrate how the selected attributes differ depending on the condition (solo or ensemble). In particular, the amount of horizontal features is predominant in the solo case whereas the vertical features are predominant in the ensemble case (Section 4.4).

\subsection{Expressive intentions}

For each expressive intention, we report the best correlation coefficients achieved for each musician and target in Table 4. The correlation coefficient of the first violin was higher than that of the other musicians most times. Another interesting phenomenon is that the correlation increased with the degree of expressivity (increasing from mechanical to exaggerated) for sound level, vibrato and bow velocity. In the case of note lengthening we did not find this trend, which suggests some relation to different tempi (the mechanical was played slower than the other two cases).

If we look at the results more closely by also considering the difference among feature sets we can draw more interesting observations: firstly, we notice that sound level and bow velocity improve on subsequent FS whereas note lengthening and vibrato stay nearly constant (see Figure 4). Secondly, we observe that the improvement, when present, follows different profiles depending on the musician and the learning task. An example of this is shown in the left plot (Sound Level) of Figure 4 (Exaggerated). The profile of the first violin distinguishes itself from the rest because it features a leap between the FS1 and FS4 and then mildly increases from FS4 to FS5. Other musicians distribute more evenly the improvement across different datasets. The profiles of each instrument are consistent across expressive intentions, and the first violin exhibits the largest improvement in correlation coefficients across feature sets.

An ANOVA on the $z$ coefficient restricted to the results of this dataset confirms our observations. We used an ANOVA model that considers the interaction effects between variables.

\begin{tabular}{|c|cccc|cccc|ccccc|}
\cline { 2 - 13 } \multicolumn{1}{c|}{} & \multicolumn{4}{c|}{ Mechanical } & \multicolumn{4}{c|}{ Normal } & \multicolumn{4}{c|}{ Exaggerated } \\
\cline { 2 - 15 } \multicolumn{1}{c|}{} & SLev & Vib & BVel & Dur & SLev & Vib & BVel & Dur & SLev & Vib & BVel & Dur \\
vl2 & $\mathbf{0 . 7 0}$ & $\mathbf{0 . 4 8}$ & $\mathbf{0 . 6 6}$ & $\mathbf{0 . 2 9}$ & $\mathbf{0 . 7 6}$ & 0.65 & $\mathbf{0 . 7 3}$ & 0.25 & $\mathbf{0 . 8 1}$ & $\mathbf{0 . 6 8}$ & $\mathbf{0 . 8 4}$ & 0.18 \\
vla & 0.46 & 0.28 & 0.50 & 0.26 & 0.46 & $\mathbf{0 . 7 0}$ & 0.53 & 0.19 & 0.60 & 0.65 & 0.67 & 0.14 \\
cello & 0.67 & 0.42 & 0.49 & 0.26 & 0.65 & 0.63 & 0.47 & $\mathbf{0 . 4 8}$ & 0.68 & 0.54 & 0.58 & $\mathbf{0 . 3 2}$ \\
& 0.44 & 0.17 & 0.49 & 0.14 & 0.69 & 0.63 & 0.61 & 0.31 & 0.76 & 0.54 & 0.80 & 0.20 \\
\hline
\end{tabular}

Table 3. Correlation coefficient on each expressive intention. Each cell contains the best correlation coefficient across feature sets for the specific musician and task. The highest value of each column is shown in bold. 
Normal
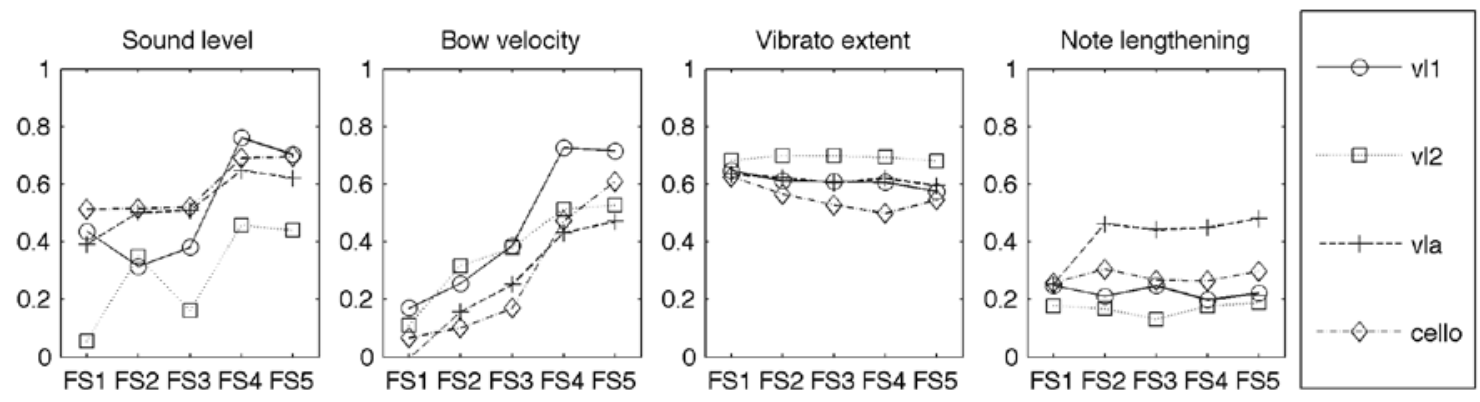

Mechanical
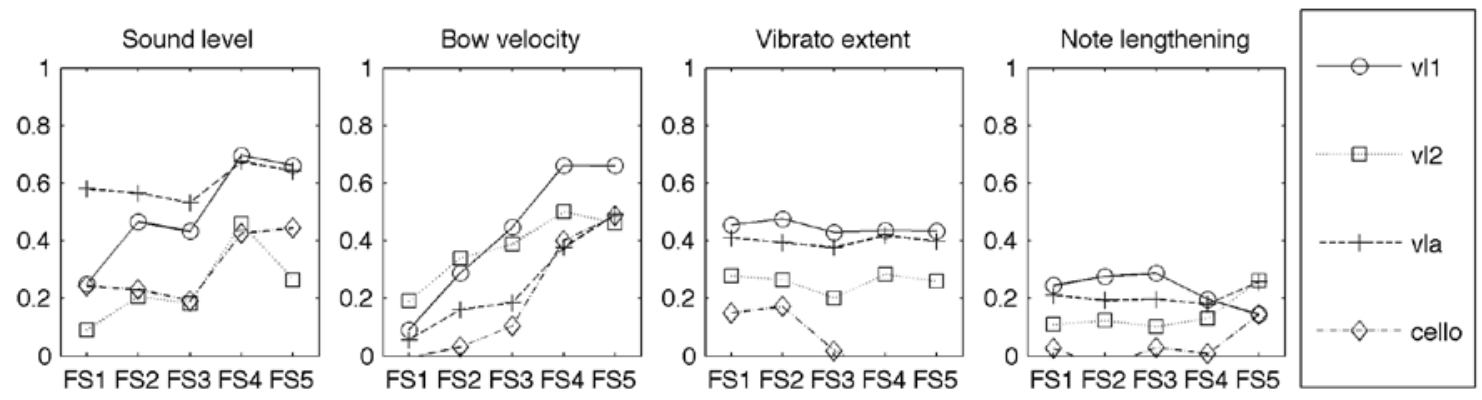

\section{Exaggerated}
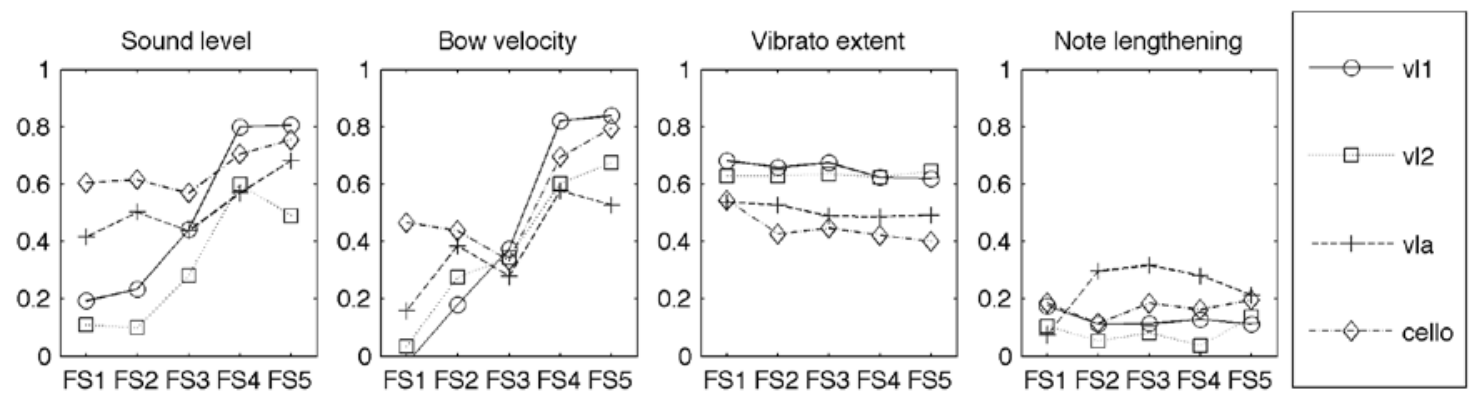

Figure 4. Resulting correlation coefficient for each expressive intentions, targets and musicians.

The factors showing a significant effect $(p<0.01)$ in order of explained variance (ss: mean sum of squares) are the following: learning task (1.60 ss), expressive intention (0.73 ss), FS (0.59 ss), FS-learning task (0.25 ss), musician (0.23 ss), learning task-intention (0.22 ss), musician-learning task (0.18 ss), musician-intention (0.12 ss), FS-musician (0.03 ss). No significant interaction between the FS and intention was found.

\subsection{Solo Vs. Ensemble}

Results for the solo vs. ensemble scenario are comparable with those obtained in the previous scenario. The main factors (in terms of explained variance) are the learning task (0.69 $\mathrm{ss})$, the interaction between musician and learning task (0.16 ss) and the FS (0.15 ss). The 


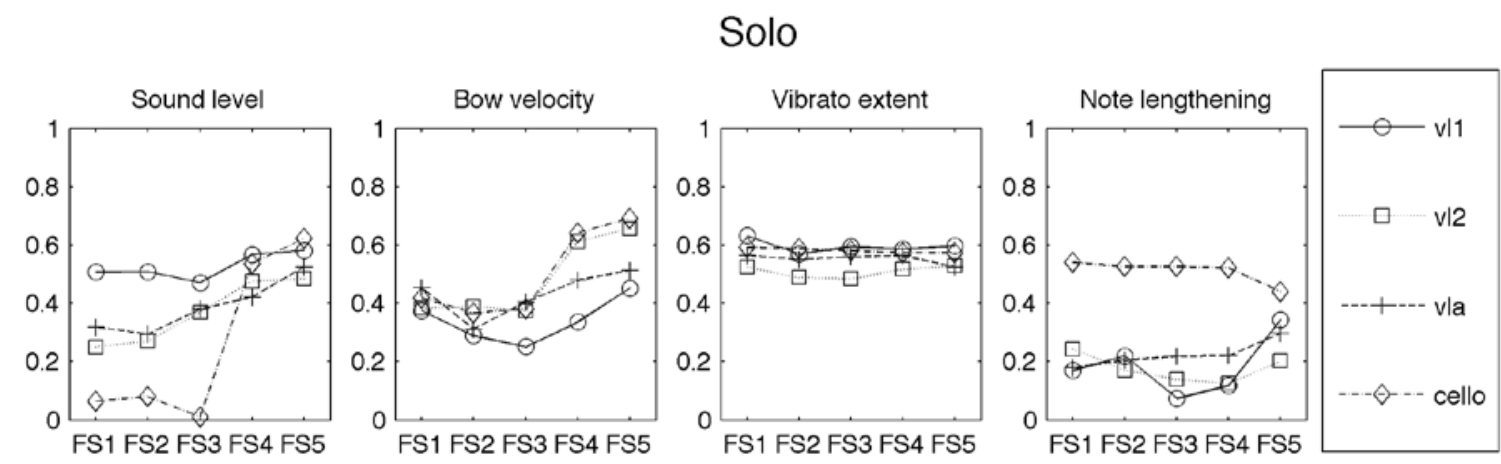

\section{Ensemble}
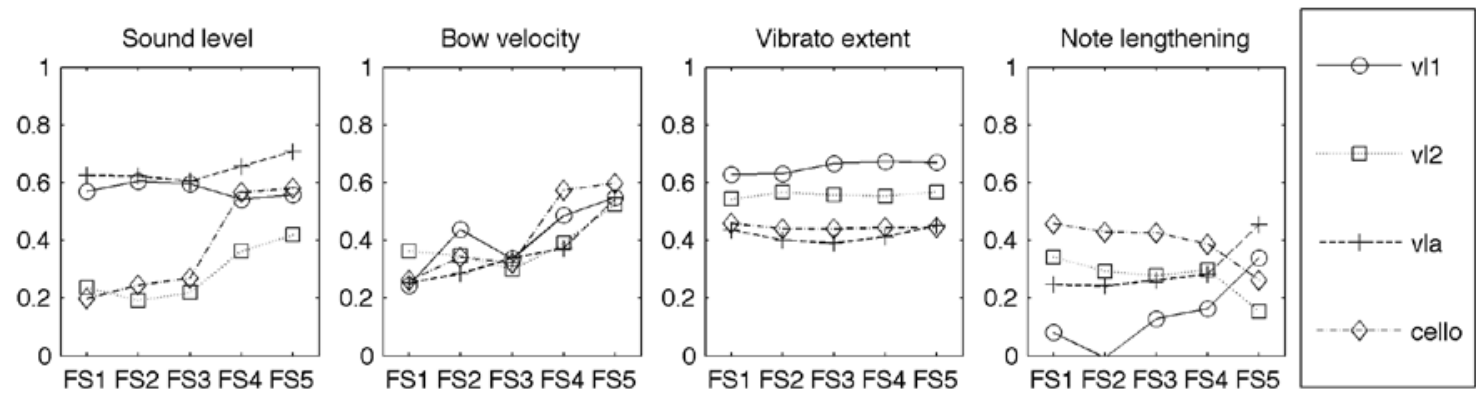

Figure 5. Resulting correlation coefficient for different learning tasks and musicians in the "solo vs. ensemble" scenario.

ANOVA analysis in this case revealed an interaction of the condition (sololensemble) with the learning task (0.06 ss) and the musician (0.04 ss). There is no evidence of any interaction between the condition and the FS $(p=0.86)$. We can, in fact, observe similar profiles of improvements in the solo and in the ensemble case (see Figure 5) for every given musician and target.

Surprisingly, on average, when adding the concurrent performance parameters of the other musicians (in FS5) there is an improvement on the prediction both in the ensemble and in solo performance (see Figure 5). The similarity between the two conditions can be explained as an effect of being accustomed to performing that material together; since the recorded excerpts were part of the ensemble's repertoire, the musicians were already accustomed to performing those excerpts together and could anticipate the actions of the rest of the ensemble even if they were not present. Nonetheless, we have to consider that the correlation profiles display no information about the underlying process of feature selection through which they were obtained. We comment on the results of feature selection in Section 4.4.

\subsection{Effect of Different ML Algorithms and Evaluation Method}

The results presented in Section 4.1 and 4.2 were obtained by removing notes in the training set which were repetitions of notes in the test set. In order to further validate our results we considered three additional factors. The first is the type of machine learning algorithm; in order to account for this factor we repeated all of the tests using the SVM and kNN instead of model 


\begin{tabular}{|l|c|c|c|c|}
\cline { 2 - 5 } \multicolumn{1}{c|}{} & \multicolumn{2}{c|}{ Solo } & \multicolumn{2}{c|}{ Ensemble } \\
\cline { 2 - 5 } \multicolumn{1}{c|}{} & PHF & p-value & PHF & p-value \\
\hline Sound Level & $54,06 \%$ & $<0,001$ & $45,26 \%$ & 0,02 \\
\hline Bow Velocity & $35,58 \%$ & 0,22 & $38,92 \%$ & 0,059 \\
\hline Vibrato Extent & $60,27 \%$ & $<0,001$ & $42,98 \%$ & 0,079 \\
\hline Note Lengthening & $52,38 \%$ & 0,007 & $23,96 \%$ & 0,173 \\
\hline
\end{tabular}

Table 4. Results of feature selection. We report the mean percentage of horizontal features (PHF) across musicians for the solo and the ensemble excerpts dataset after the feature selection. Notice how PHF is higher in the solo than in the ensemble case (except for bow velocity). We report the one-tailed $p$-values for each percentage.

trees. The second factor is the influence of repetitions in the dataset arising from refrain marks in the score. We wanted to discover what happens if we do not remove the repetitions, and so repeated all the tests without this correction. The third factor was to use a 10-fold cross validation instead of using phrases as folds. In a 10-fold cross validation, instead of using the phrases to build the folds we randomly split the dataset into 10 subsets, which are used, one at a time, as test sets.

We ran an ANOVA on all $z$ values, considering the classifier, the removal of repeated notes and the evaluation method among the factors. All three effects were, alone, found significant (with $\mathrm{p}<0.03$ ). In particular, the cross-validation per phrase yielded lower values of correlation with respect to a simple 10-fold cross-validation. Regarding the effect of the classifier, the model trees provided best results, followed, in order, by SVM and kNN. Removing the repetitions also significantly lowered the correlation coefficients, which was expected from the resulting reduction in size of the training sets. We found interaction between classifier and the effect of removing repetitions $(p<0.03)$. Indeed, the effect of removing repetitions was significant (the mean correlation dropped by 0.10 ) in the case of kNN whereas both SVM and model trees were less affected (for both the mean correlation dropped by 0.04). This confirms that removing repetitions was important for guaranteeing that there is no over fitting, especially for the case of kNN. Additionally, we can conclude that model trees and SVM are not reliant on those few repeated instances as much as kNN is and are thus more reliable for building a robust model of expressivity.

\subsection{Analysis of Feature Selection on the Solo vs. Ensemble scenario}

On each of the setups we computed the difference between the correlation coefficient after and before applying feature selection. The mean of such differences across all the tests was 0.03 although this value became higher in the models employing larger feature sets. This means that the more features there are, the better the improvement we can achieve by applying feature selection. By considering the difference in terms of $z$ coefficients we also performed a ttest, which showed that this average difference, is significantly positive $(p<0.01)$. We can deduce that the feature selection procedure improves the prediction.

The totality of 67 features is divided into 21 horizontal features (all the attributes of Table 1 plus 2 expressive performance values attributes) and 46 vertical features (all the attributes of 
Tables 2 and 3 plus 6 expressive performance values attributes). The percentage of horizontal features was therefore $31.3 \%$ in the FS5. We analyzed how this percentage changed after the feature selection in the solo vs. ensemble scenario.

Table 5 reports the mean across musicians of the percentage of horizontal features (PHF) for each learning task and condition. Overall, we can observe how feature selection tends to select horizontal and vertical features more uniformly. The horizontal features were in fact relatively more numerous after feature selection with respect to what they were in the full FS5. We computed p-values for each percentage based on the null-hypothesis that feature selection is modeled by an urn extraction without replacement. This statistic is thus based on a sample percentage mean computed over the results of independent draws with hyper-geometric distributions. We report one-tailed p-values for each mean percentage. Small p-values $(<0.05)$ indicate that the obtained percentage cannot be attributed to chance. PHF is generally higher in the solo than in the ensemble. In the case of solo, percentages were higher than $50 \%$ (except for bow velocity), which is very unlikely, given that by pure chance the expected percentage should be close to the original $31.3 \%$. In the case of sound level, vibrato extent and note lengthening the PHFs are large enough to reject the null hypothesis that they arise from chance (see second column of solo in Table 5). In the case of ensemble, the percentages are all lower than $50 \%$ and thus more vertical features were selected.

\section{Discussion}

The analysis of feature selection exhibited a clear tendency of the models to prefer horizontal features (individual voice context) in the solo case, and to prefer vertical features (inter-voice relation context) in the ensemble case. This confirms the validity of the introduced inter-voice features in the context of ensemble expressive performance.

In light of the obtained results, we offer some thoughts on how they can be explained from a musical standpoint involving the musicians and the listeners. The average difference in predictive power among expressive parameters suggests different management of each nuance. Sound level and bow velocity are related to expressive amplitude variations. The increase in predictive power towards models with representation of inter-voice context implies that the intervoice features helped the models. The fact that this improvement can be measured both in solo and ensemble recordings suggests that even when a player is playing solo, they have in their minds the imagined parts of the other members of the quartet. Also, for the listener, expressive use of amplitude variation will simply not be heard if masked by other instruments playing at the same time, so this is highly dependent on simultaneous notes. In the case of vibrato, we believe that, since musicians know from experience that it is more difficult to perform a vibrato in short notes, they might reserve it for notes that are longer. As a result, vibrato extent might be already well predictable based solely on the individual voice. In the case of note lengthening the results were, instead, not sufficient to prove a substantial advantage of using neither horizontal nor vertical descriptors. This suggests that, in the case of timing, the excess of timing unrelated features corrupted the performance of the models and, thus, simpler models based on time keeping correction mechanisms (Repp, 2005) and phrasing (Widmer \& Tubudic, 2003) might still be more suited. However, it is still possible that if trained on larger datasets this method might autonomously discover such synchronization mechanisms and achieve better predictions. 
We noticed that the predictive power of the models tends to improve when musicians play more expressively. Differences in predictive power across musicians tend to favor the first violin as the musician whose expressivity is easier to predict in terms of sound level, bow velocity and vibrato extent. We believe that this tendency is a good sign, since it means that the model is really modeling the intentional expressivity employed by the musicians, and not some perceptually irrelevant byproduct of playing an instrument.

\section{Conclusion}

We introduced an approach to modeling expressive performance in string quartets allowing for polyphonic expression and inter-dependence among voices. We recorded string quartet performances and extracted a set of expressive parameters related to sound properties of each performed note by exploiting multi-track audio data and bowing motion data. We assigned an expressive model to each musician and trained it on the collected data using supervised machine learning algorithms. We gradually instilled into the models the ability to represent score and performance inter-voice relations by incorporating in the feature set of the models a number of ensemble-specific contextual descriptors. We evaluated how well models built on different feature sets were fit for predicting the expressive performance data by quantifying their predictive power by means of a cross validation scheme.

Difference between models trained on solo condition and models trained in ensemble condition was found in terms of feature selection. From one side, this means that the models captured the different nature of the two performing scenarios by representing the musical knowledge of how to play expressively in terms of different features: predominantly horizontal/individual voice features for solo performance, predominantly vertical inter-voice features for ensemble performance. On the other side, this also confirmed the validity of the introduced vertical features in the context of ensemble performance modeling. However, the fact that the predictive power of both solo and ensemble models improved when adding inter-voice features suggests that subtler correlations among the solo performances remained. We speculated that this residual correlation might be derived from the established practice of the string quartet members to play together that piece. We thus suggest future experiments in which musicians record their solo part before having rehearsed it in ensemble.

One main limitation of this work was given by the absence of databases with annotated ensemble recordings and this is why one of our contributions was to carry out multimodal ensemble recording of string quartets. We believe that on larger datasets the presented method could reveal even more in-depth information about musicians' roles and music interaction in ensembles allowing a comparison among different music styles and different quartets. Furthermore, on larger dataset comprising the performance of a number of different pieces, the

obtained models could be easily applied to the generation of polyphonic expression in ensemble music.

\section{Acknowledgments}

This work was partially supported by the EU FP7 FET-Open SIEMPRE Project no. FP7-ICT2009-C-250026 and by the Catalan Government. We would also like to thank Erika Donald, 
Alfonso Pérez and Marcelo Wanderley for the support in organizing the experiment, as well as CIRMMT and BRAMS labs at Montreal, Quebec, Canada for hosting them. We thank Rafael Caro for helping us with the musicological analysis.

\section{References}

Askenfelt, A. (1986). Measurement of bow motion and bow force in violin playing. Journal of the Acoustical Society of America, 80(4):1007-1015.

de Cheveigne, A., \& Kawahara, H. (2002). YIN, a fundamental frequency estimator for speech and music. Journal of the Acoustical Society of America, 111(4):1917-1930.

Cristianini, N., \& Shawe-Taylor, J. (2000). An introduction to Support Vector Machines and Other Kernel-based Learning Methods. Cambridge University Press.

Friberg, A., (1995). A Quantitative Rule System for Musical Performance. PhD thesis, Royal Institute of Technology, Stockholm, Sweden.

Gabrielsson, A. (1999). The performance of Music. In D.Deutsch (Ed.), The Psychology of Music (2nd ed.) Academic Press.

Gabrielsson, A. (2003). Music Performance Research at the Millennium. Psychology of Music, Vol. 31, No. 3, 221-272 (2003)

Goebl, W., \& Palmer, C. (2009). Synchronization of Timing and Motion Among Performing Musicians. Music Perception: An Interdisciplinary Journal, Vol. 26, No. 5, pp. 427-438

Hall, M. A. (1998). Correlation-based Feature Subset Selection for Machine Learning. PhD thesis, Hamilton, New Zealand.

Hall, M., Frank, E., Holmes, G., Pfahringer, B., Reutemann, P., \& Witten, I. H. (2009). The WEKA Data Mining Software: An Update; SIGKDD Explorations, Volume 11, Issue 1.

Herrera, P. (1998). Vibrato extraction and parameterization in the Spectral Modeling Synthesis framework. In Proceedings of the Conference on Digital Audio Effects (DAFx '98), Barcelona, Spain.

Keller, P. E. (2008). Joint action in music performance. Emerging Communication, 10, 205. IOS press.

Kendall, R.A. \& Carterette, E. (1990). The Communication of Musical Expressions. Music Perception: An Interdisciplinary Journal 8(2), pp. 129-163

Kirke, A. \& Miranda, E.R. (2013). An overview of computer systems for expressive music performance. In Guide to Computing for Expressive Music Performance, pages 1-47. Springer.

Lopez de Mantaras, R. \& Arcos, J.L. (2002). Al and music, from composition to expressive performance, Al Magazine, $\quad-33$

Maestre, E. (2009), Modeling instrumental gestures: an analysis/synthesis framework for violin bowing. PhD thesis, Universitat Pompeu Fabra, Barcelona, Spain.

Marchini, M., P. Papiotis, A. Pérez, \& E. Maestre (2011). A hair ribbon deflection model for low-intrusiveness measurement of bow force in violin performance. In New Interfaces for Musical Expression Conference.

Marchini, M., Ramirez, R., Papiotis, P. \& E. Maestre (2013). Inducing Rules of Ensemble Music Performance : A Machine Learning Approach. In Proceedings of the Third Conference on Music and Emotions (ICME 2013), Jyväskylä, Finland 
Moore, G. P., \& Chen, J. (2010). Timing and interactions of skilled musicians. Biological Cybernetics, 103, 401-414

Narmour, E. (1990) The Analysis and Cognition of Basic Melodic Structures: The ImplicationRealization Model. Chicago: University of Chicago Press.

Palmer, C. (1997). Music performance. Annual Review of Psychology, 48, 155-138.

Poli, G. D. (2004). Methodologies for expressiveness modelling of and for music performance. Journal of New Music Research, 33(3), 189-202.

Prame, E. (1997). Vibrato extent and intonation in professional Western lyric singing. The Journal of the Acoustical Society of America, 102, 616.

Ramirez, R., \& Hazan, A. (2006). A Tool for Generating and Explaining Expressive Music Performances of Monophonic Jazz Melodies, International Journal on Artificial Intelligence Tools 15(4) (2006), 673-691.

Repp, B. H. (2005), Sensorimotor synchronization: A review of the tapping literature, Psychonomic Bulletin \& Review, 12 (6), 969-992.

Sundberg, J., Friberg, A., \& Frydén, L. (1989). Rules for automated performance of ensemble music, Contemporary Music Review, Vol. 3, pp. 89-109.

Widmer, G. (2002). Machine discoveries: A few simple, robust local expression principles. Journal of New Music Research, 31(1), 37-50.

Widmer, G., \& Tubudic, A. (2003). Playing Mozart by analogy: Learning multi-level timing and dynamics strategies. Journal of New Music Research, 32, pp. 259-268.

Widmer, G., \& Goebl, W. (2004). Computational Models of Expressive Music Performance: The State of the Art. Journal of New Music Research, Vol. 33, No. 3, pp. 203-216. 